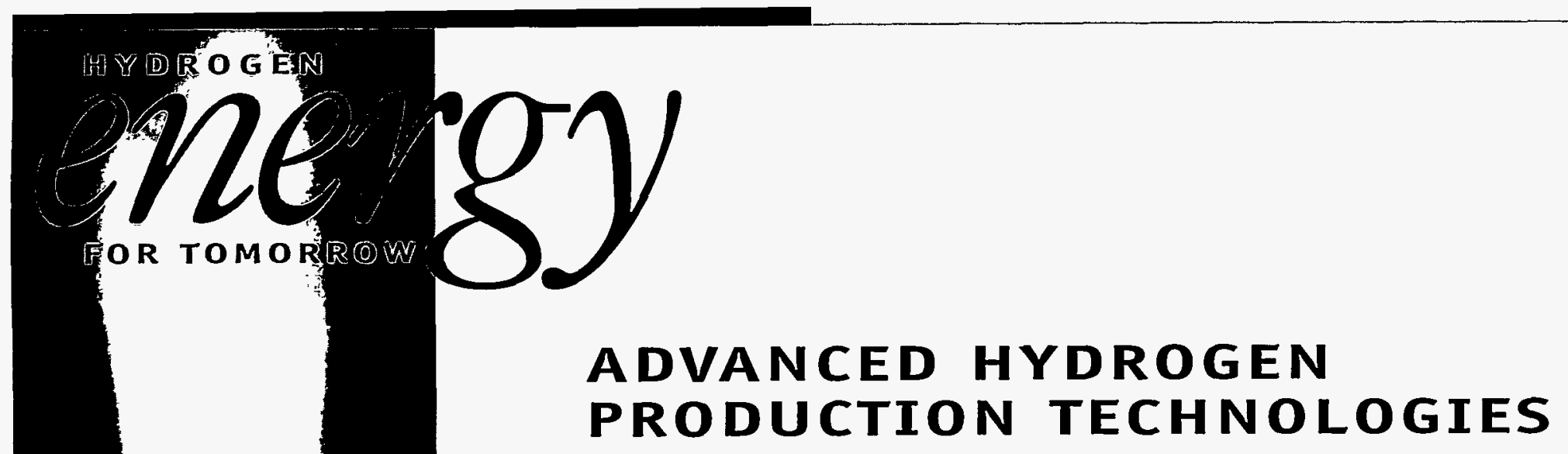

The future vision for hydrogen is that it will be cost-effectively produced from renewable energy sources and made available for widespread use as an energy carrier and a fuel. Hydrogen can be produced from water and when burned as a fuel, or converted to electricity, joins with oxygen to again form water. It is a clean, sustainable resource with many potential applications, including generating electricity, heating homes and offices, and fueling surface and air transportation.

To achieve this vision, researchers must develop advanced technologies to produce hydrogen at costs competitive with fossil fuels, using sustainable sources. Hydrogen is now produced primarily by steam reforming of natural gas. For applications requiring extremely pure hydrogen, production is done by electrolysis. This is a relatively expensive process that uses electric current to dissociate, or split, water into its hydrogen and oxygen components.

Technologies with the best potential for producing hydrogen to meer future demand fall into three general process categories: photobiological, photoelectrochemical, and thermochemical. Photobiological and photoelectrochemical processes generally use sunlight to split water into hydrogen and oxygen. Thermochemical processes, including gasification and pyrolysis systems, use heat to produce hydrogen from sources such as biomass and solid waste.

\section{PHOTOBIOLOGICAL PRODUCTION}

Most photobiological systems use the natural activity of bacteria and green algae to produce hydrogen: chlorophyll absorbs sunlight and enzymes use the energy to dissociate hydrogen from water. Photobiological technologies hold great promise for long-term sustainable hydrogen production but face at least two significant limitations.

First, they have relatively low solar conversion efficiencies, generally converting about $5 \%$ to $6 \%$ of the sun's energy to hydrogen energy. Second, nearly all enzymes that evolve hydrogen from water are inhibited in their hydrogen production by the presence of oxygen, the other key product of water dissociation.

Researchers are addressing both of these limitations with projects involving whole-cell organismsbacteria and green algae-and cell-free systems composed of enzymes taken from microorganisms. Research in these technologies is in the early stages and is focusing on overcoming oxygen intolerance and increasing conversion efficiencies. 


\section{Biological Production in the Dark}

Photobiological production of hydrogen generally involves energy from sunlight, but a unique type of hydrogen-producing activity has been identified in certain strains of cyanobacteria. Nearly 400 strains have been identified that can combine carbon monoxide ( $\mathrm{CO}$ ) and water $\left(\mathrm{H}_{2} \mathrm{O}\right)$ in darkness and produce hydrogen and carbon dioxide at very high production rates. Twenty-five of these bacteria strains have been examined in some detail. Impressively, all 25 produce hydrogen from nearly $100 \%$ of the $\mathrm{CO}$ at ambient temperature and pressure.
In one effort to overcome oxygen intolerance, strains of bacteria have been identified with hydrogen-evolving enzymes more tolerant of oxygen than those in most bacteria. The enzymes have been extracted from one of these strains and genetically introduced into a green algae-the Cblamydomonas strain-that has oxygen-evolving enzymes. This has created new algal strains that produce hydrogen and oxygen simultaneously.

Cell-free processes are another effective means of solving the problem of oxygen sensitivity in these organisms. In a cell-free system, both oxygen-evolving and hydrogen-evolving enzymes are immobilized onto opposite sides of a solid, conducting surface. Light is used by one enzyme to oxidize water, creating a flow of electrons to the other enzyme, where the hydrogen is produced. Energy conversion efficiency of a cell-free system could theoretically approach $25 \%$.

A leading effort to increase conversion efficiencies also involves the green alga Chlamydomonas and focuses on the two reaction sequences in green plants that use light to synthesize carbohydrates and hydrogen. A pigment system in one sequence directly splits water, which produces oxygen and creates a flow of electrons to the other sequence. The second sequence uses the electrons to reduce carbon dioxide to carbohydrates. The electrons can also be used to produce hydrogen when the organism is in an oxygen-free environment.

New genetic forms of Chlamydomonas have been creared that possess only the water-splitting reaction sequence. These strains are able to sustain simultaneous production of hydrogen and oxygen using only one light reaction, substantially increasing the alga's potential conversion efficiency. Cblamydomonas with both reaction sequences has solar conversion efficiencies of about $10 \%$; with a single light reaction this could, theoretically, be doubled to about $20 \%$.

\section{PRODUCTION BY PHOTOELECTRO- CHEMICAL TECHNOLOGY}

Photoelectrochemical (PEC) production uses semiconductor technology in a one-step process of splitting water directly upon sunlight illumination. A PEC system combines a photovoltaic cell-material that produces electric current when exposed to light-and an electrolyzer in a single device. This process has shown excellent potential for producing low-cost renewable hydrogen.

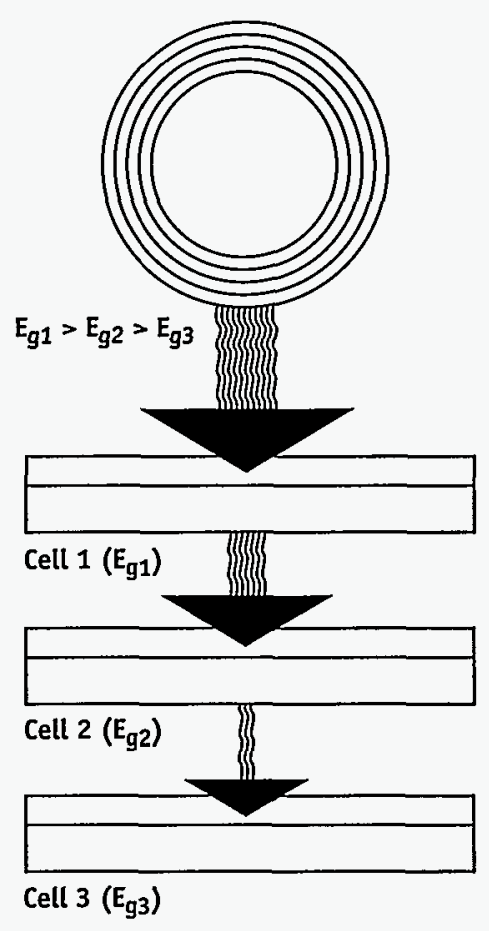

A multijunction device stacks individual pbotovoltaic cells that accept different solar energy levels $(E g)$ on top of each otber; a configuration that converts more of the energy spectrum of light to electricity. Sunlight falls first on the material capturing the bigh-energy protons and passes the rest of the protons on to be absorbed by the lower cells. 


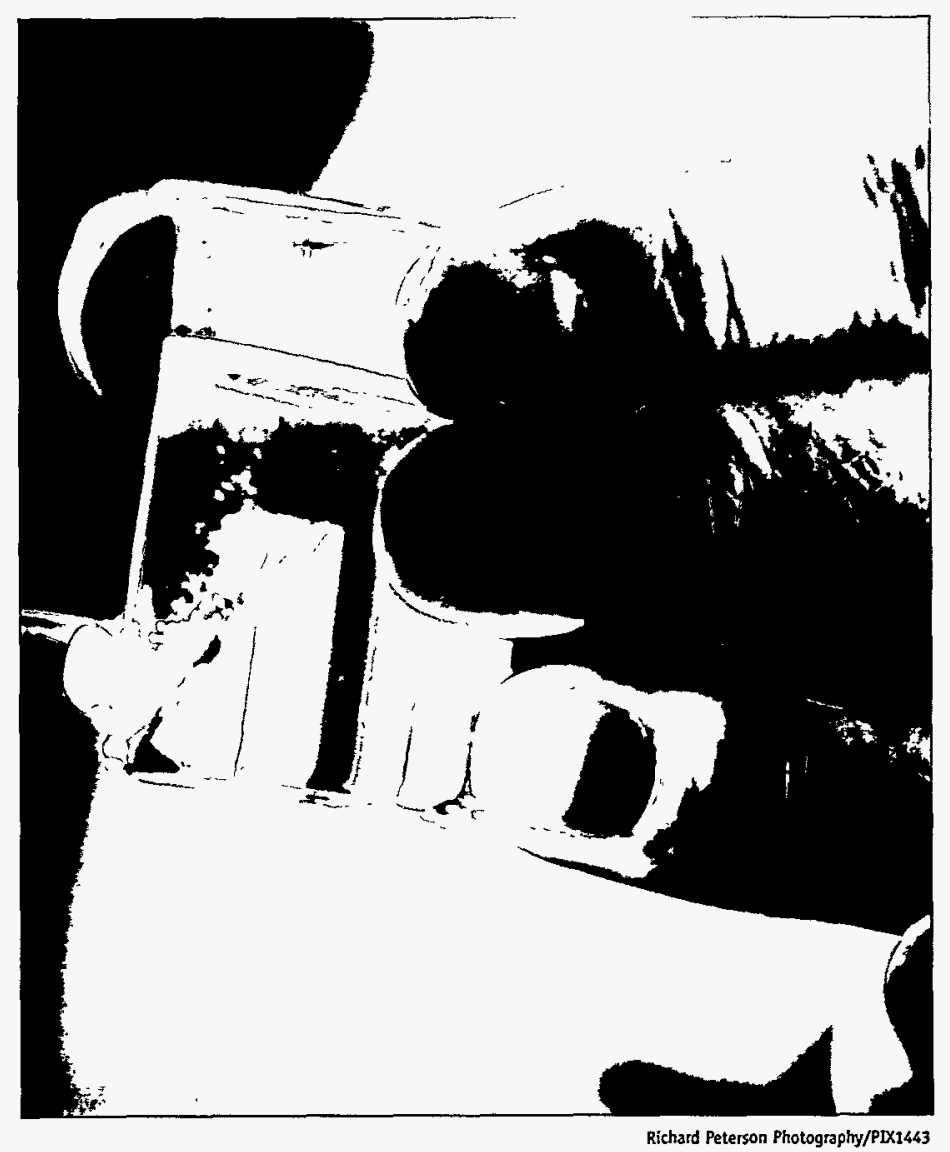

Hydrogen and oxygen bubbles emanate from a combined photovoltaic cell and electolyzer submerged in an alkaline aqueous solution. The penlight on the cell generates enough voltage to dissociate the water in the solution. The surface of the photovoltaic cell is coated with a metal catalyst that acts as an electrolyzer.

The most promising materials for PEC hydrogen systems are amorphous silicon, amorphous silicon carbide, gallium indium phosphide $\left(\mathrm{GaInP}_{2}\right)$, and gallium arsenide ( $\mathrm{GaAs}$ ). These types of photovoltaic materials can provide the higher voltages required for electrolysis and have relatively high solar efficiencies. A GaInP 2 multijunction cell has achieved a solar conversion efficiency of more than $25 \%$, and amorphous silicon devices have achieved efficiencies of greater than $13 \%$.

Gallium-based cells are very expensive, while amorphous silicon solar cells are designed for low cost. Researchers are working to combine low materials costs and high conversion efficiencies to achieve a practical application of this promising technology.

\section{THERMOCHEMICAL PRODUCTION}

Progress in developing an efficient and cost-effective PEC system has primarily been limited by two factors: (1) the high voltage required to dissociate water and (2) the corrosiveness of the aqueous electrolytes used in the electrolytic process. Keys to overcoming these limits are the structure and materials of the photovoltaic device.

Photovoltaic systems can be structured in either single-cell or multijunction devices, consisting of two or three cells stacked on one another. The multijunction design can use the energy of a larger fraction of the solar spectrum of light than a single-cell unit. This makes it more effective at producing the higher energy-greater than 1.6 electron volts-required to split water.
The gasification and pyrolysis methods of hydrogen production use heat to produce a vapor from which hydrogen can be derived using a conventional steam reforming process. Using biomass-wood, grasses, and agricultural and municipal waste - as a feedstock for these processes provides a sustainable source for hydrogen production.

In pyrolysis, biomass is broken down into highly reactive vapors and a carbonaceous residue, or char. The vapors, when condensed into pyrolysis oils, can be steam reformed to produce hydrogen. A typical biomass feedstock produces about $65 \%$ oils and $8 \%$ char by weight, with the remainder consisting of water and gas. 


\section{Gasification under Water}

A unique approach to hydrogen production is the gasification of high-moisture aquatic plants. Using aquatic crops for gasification is desirable because their cultivation doesn't compete with land food production. A major drawback to the use of conventional combustion processes, however, has been the high cost of drying the plants.

The potential for this process has been shown with the successful gasification of wet biomass in supercritical water at relatively low temperatures. This was completed at $600^{\circ} \mathrm{C}$ and 34.5 megapascals in a 30-second reaction time using a catalyst.

The product gas contained hydrogen, carbon dioxide, carbon monoxide, methane, ethane, propane, and other hydrocarbons. The hydrogen yield was high; most other products can be converted to hydrogen with conventional steam reforming.

The char is burned to provide the required process heat for the pyrolysis reaction. For hydrogen production, a fast-pyrolysis reactor is directly linked to a steam reformer. Such processes have yielded from $12 \%$ to $17 \%$ hydrogen by weight of dry biomass. This technology could be one of the lowestcost production methods; researchers are focusing on identifying optimum reformer catalysts.

The gasification of municipal solid waste (MSW) is a potentially low-cost, sustainable source of hydrogen production. MSW, on the average, consists of about $70 \%$ by weight of biomass material. Gasification results in an easily cleaned fuel gas from which hydrogen can be reformed.

To develop a practical MSWto-hydrogen system, researchers are evaluating Texaco's high-temperature gasification process for converting from fossil fuels to biomass feedstock. This process results in a high yield of hydrogen and produces a nonhazardous, glass-like ash byproduct.
The Texaco system can be adapted to MSW by adding high-pressure hot warer (hydrothermal) preprocessing for slurry preparation. Actual MSW samples-as opposed to laboratorysimulated waste-will be tested in a modified Texaco hydrothermal preprocessing pilot plant to establish favorable processing conditions.

\section{CONCLUSION}

These are just a few of the many technologies and research projects involving hydrogen production. Work continues to develop viable production processes that will support the hydrogen energy economy of the future. To learn more about hydrogen production technologies or to inquire about opportunities for your company to be involved in the U.S. Department of Energy's cooperative research programs, please contact:

\section{U.S. DEPARTMENT OF ENERGY Neil Rossmeissl, Hydrogen Program Manager Telephone: (202) 586-8668 Fax: (202) 586-0784}

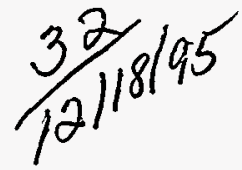

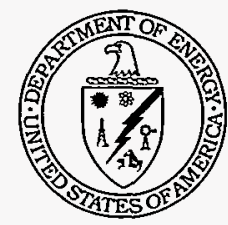

Produced for the

U.S. DEPARTMENT OF ENERGY 1000 Independence Avenue, SW Washington, DC 20585

by the

\section{NATIONAL RENEWABLE ENERGY LABORATORY}

a DOE national laboratory

DOE/GO-10095-065

DE95000270

August 1995 